\title{
GT-2002-30500
}

\section{A COMPUTER CODE FOR GAS TURBINE ENGINE WEIGHT AND DISK LIFE ESTIMATION}

\author{
Michael T. Tong \\ NASA Glenn Research Center \\ Cleveland, Ohio
}

\author{
Ian Halliwell \\ Modern Technologies Corporation \\ Cleveland, Ohio
}

\author{
Louis J. Ghosn \\ Ohio Aerospace Institute \\ Cleveland, Ohio
}

\begin{abstract}
Reliable engine-weight estimation at the conceptual design stage is critical to the development of new aircraft engines. It helps to identify the best engine concept amongst several candidates. In this paper, the major enhancements to NASA's engine-weight estimate computer code (WATE) are described. These enhancements include the incorporation of improved weight-calculation routines for the compressor and turbine disks using the finite-difference technique. Furthermore, the stress distribution for various disk geometries was also incorporated, for a life-prediction module to calculate disk life. A material database, consisting of the material data of most of the commonly-used aerospace materials, has also been incorporated into WATE. Collectively, these enhancements provide a more realistic and systematic way to calculate the engine weight. They also provide additional insight into the design trade-off between engine life and engine weight. To demonstrate the new capabilities, the enhanced WATE code is used to perform an engine weight/life trade-off assessment on a production aircraft engine.
\end{abstract}

Keywords: Engine Weight, Finite Difference, Disk Life

\section{INTRODUCTION}

Engine weight is a key design parameter for any new aircraft engine. It affects aircraft range and is a key element in fuel burn. Weight is also considered an indicator of engine cost. Reliable engine-weight estimation at the conceptual design stage is critical to the development of new aircraft engines. It helps to identify the best engine concept amongst several candidates.

Equally important, aircraft engines must meet safety demands. Fatigue loading of turbine components associated with continuous aircraft take-off/cruise/landing cycles is a principal source of degradation in turbomachinery. A disk burst is potentially the most catastrophic failure possible in an engine and thus disks are designed with overspeed capability and low cycle fatigue life as primary objectives. The requirement for higher turbine stage work without additional stages has resulted in increased turbine blade tip speeds and higher turbine inlet temperatures in advanced commercial aircraft engines. This trend has resulted in significant increases in turbine stage disk rim loading and a more severe thermal environment, thereby making it more difficult to design turbine disks for a specific life requirement meeting current goals. Current trend indicates that both turbine blade tip speeds and turbine inlet temperatures will continue to increase in advanced commercial engines as higher turbine work levels are achieved. Advanced turbine disk concepts are required to insure long life disks in commercial engines, without resulting in severe weight, performance, or cost penalties.

At NASA Glenn Research Center (GRC), the Weight Analysis of Turbine Engines (WATE) computer code [1], originally developed by Boeing Aircraft, is currently used to estimate the engine weight of various conceptual engine designs. The code was originally developed for NASA in 1979, but since then, substantial improvements have been made to the code to improve the weight calculations for most of the engine components. Recently a series of efforts were performed at GRC to enhance the capability of the WATE code. In this paper, these WATE code enhancements are described. The major enhancements include the incorporation of improved weightcalculation routines for the compressor and turbine disks using the finite-difference technique. Furthermore, the stress distribution for various disk geometries was also incorporated, for a life-prediction module to calculate disk life. A material database, consisting of the material data of most of the commonly-used aerospace materials, has also been incorporated into WATE. Collectively, these enhancements provide a more realistic and systematic way to calculate the engine weight. They also provide additional insight into the design trade-off 
between engine life, weight, and cost. The current effort paves the way for an automated engine design tool, which would easily allow engine developers to perform design trade-offs between engine performance, durability, and cost. To demonstrate the new capabilities, the improved WATE code is used to perform an engine weight/life trade-off assessment on a production aircraft engine.

\section{NOMENCLATURE}

D true tensile ductility $=\ln \left[\frac{(\% R A)}{(100-\% R A)}\right]$

E modulus of elasticity

$\mathrm{N}_{\mathrm{f}} \quad$ cycles to fatigue crack initiation failure

RA reduction in area at failure

$T_{0} \quad$ reference temperature $=$ room temperature

$T_{\text {bore }} \quad T_{\max }-\Delta T$

$T_{\max } \quad$ maximum disk metal temperature, assumed to be at

the rim; user input

$\Delta T \quad$ temperature difference between disk rim and bore; user input

$c \quad$ axial chord at the blade hub

$n_{b} \quad$ number of blades

$m_{b} \quad$ mass of one blade

$r_{c g} \quad$ radius of the c.g. of the dead weight

$r \quad$ radial distance

$\mathrm{r} 1, \mathrm{r} 2, . . \mathrm{r} 6$ various radial location along the disk

$r_{l} \quad$ outer radius of the live disk

$r_{i} \quad$ inner radius (disk bore)

$r_{o} \quad$ outer radius (disk rim)

$\Delta r \quad$ change in radius

sf $\quad 1.1$, safety factor

$t \quad$ local disk thickness at $r$

$\Delta t \quad$ change in disk thickness

$u \quad$ radial displacement

$\rho \quad$ disk material density

$\omega \quad$ rotational speed, $(\mathrm{rad} / \mathrm{sec})$

$\alpha \quad$ coefficient of thermal expansion

$v \quad$ Poison's ratio

$\sigma_{y} \quad$ yield strength of the disk material at the local

temperature

$\sigma_{e} \quad$ equivalent or Von Mises stress

$\sigma_{\theta a v g} \quad$ average tangential stress of the disk

$\sigma_{u t s} \quad$ ultimate tensile strength

$\sigma_{r} \quad$ radial stress
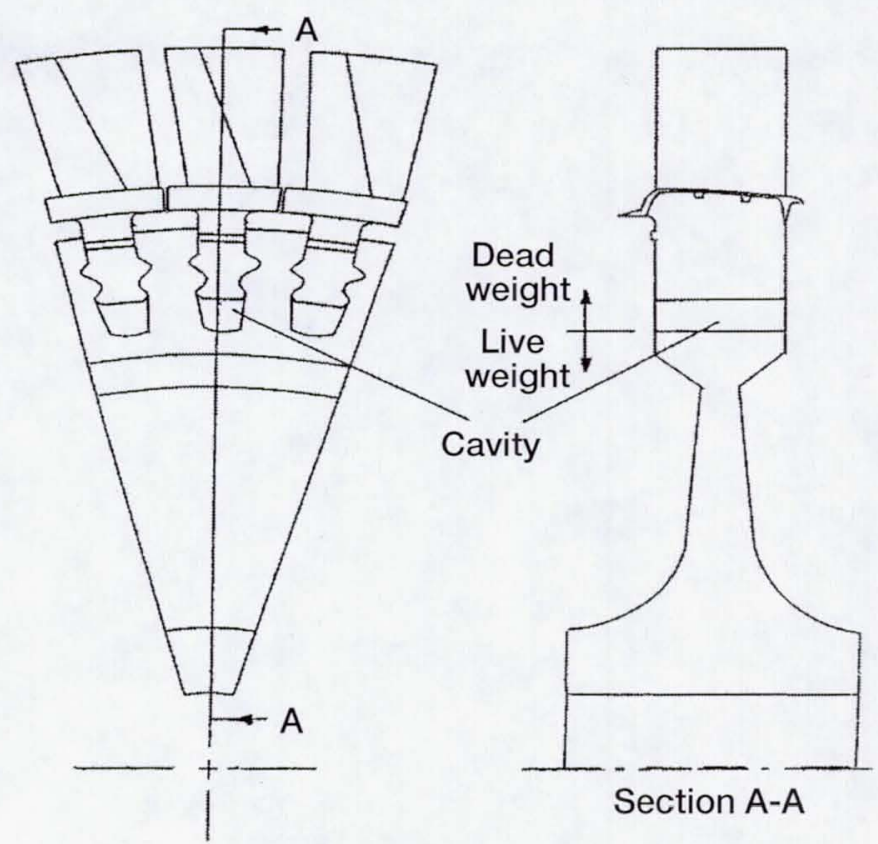

Figure 1.-Disk/blade assembly.

$\begin{array}{ll}\sigma_{\theta} & \text { tangential stress } \\ \Delta \varepsilon_{t} & \text { total strain range } \\ \Delta \varepsilon_{e l} & \text { elastic strain range } \\ \Delta \varepsilon_{\text {in }} & \text { inelastic strain range }\end{array}$

\section{OBJECTIVE}

The objective of the current work is to enhance the current engine flow-path design tool so that it will allow engine developers to easily perform design trade-offs between engine weight, durability, and eventually, cost.

\section{TURBOMACHINERY DISK DESIGN METHODOLOGY}

A typical disk design is based on the blading geometry, weight, and rotational speed that will already have been specified in the design of the turbomachinery flowpath. Figure 1 shows how blades are typically attached to a disk, and introduces some of the nomenclature. The typical three disk models (ring, web, and hyperbolic) are shown in figures 2 to 4 . The flowpath height or blade span is defined via the blade tip and hub radii, which are primary inputs to the WATE code. The disk rim thickness is assumed to be equal to the axial chord at the hub of the blade. The height of the blade root is also specified within the program, as a percentage of the airfoil height, and it defines the outer limit of the live disk. Note that some allowance must be made for the cavity, since this is not modeled specifically. Moving inwards towards the centerline, additional rim height is then required to support the stresses generated by the dead weight, and this is specified as a function of the rim thickness. Regardless of the disk type, the distribution of thickness for the remainder of the disk is specified initially by minimum default values at key radial 


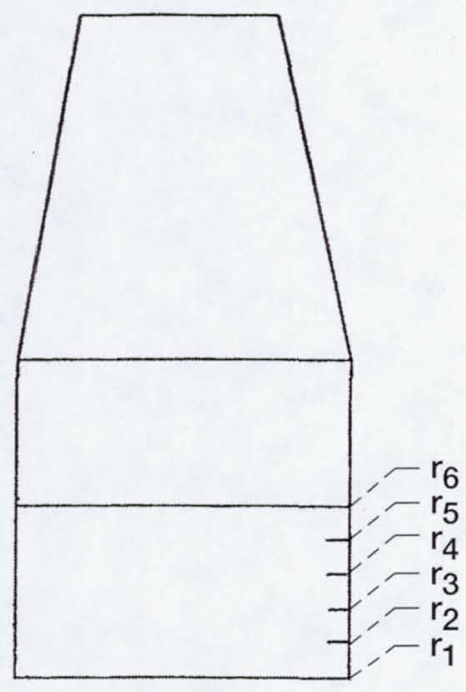

$t_{1}=t_{2}=t_{3}=t_{4}=t_{5}=t_{6}$
Airfoil

Blade root

+ Post

Outer rim

Outer Shoulder

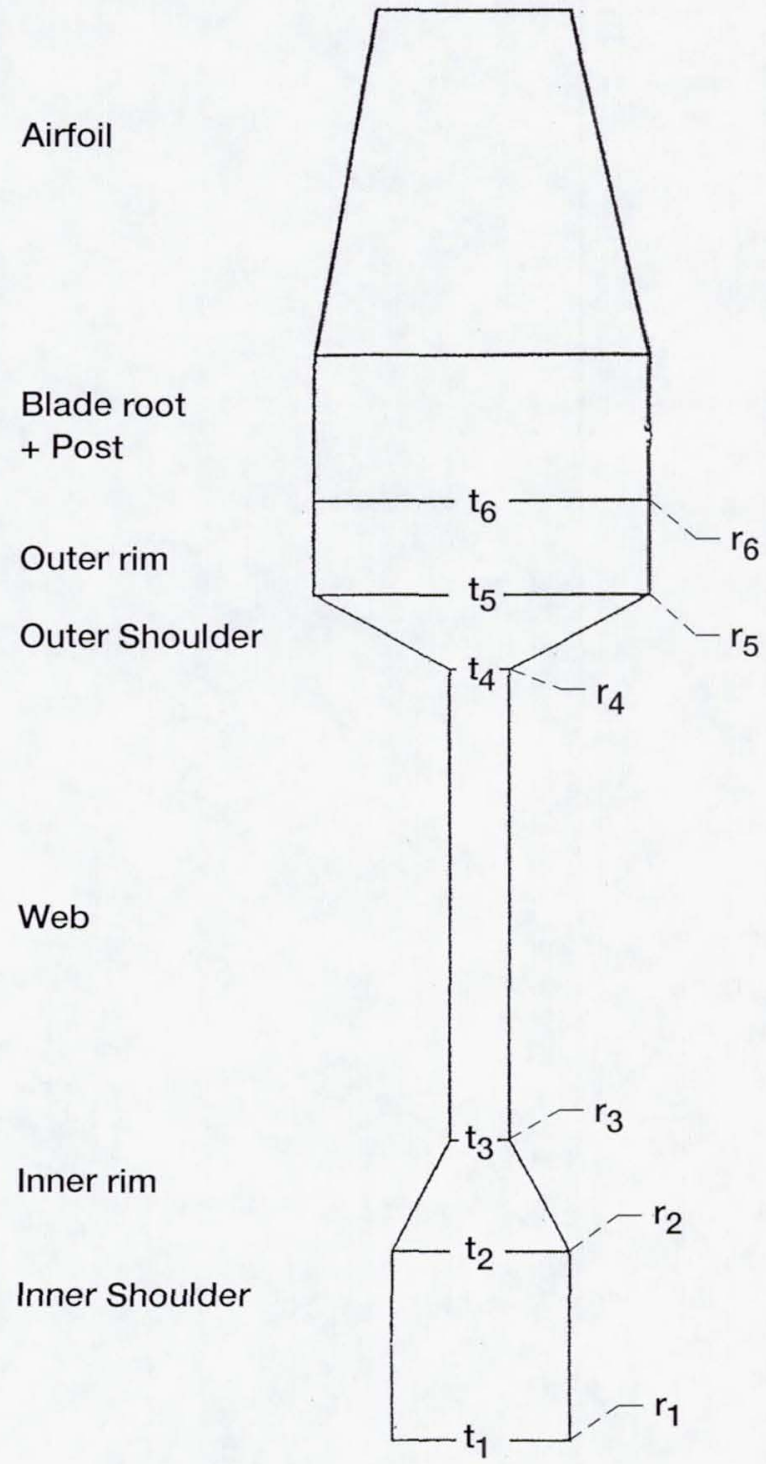

Web

Figure 2.-Radial stations and locations of design control calculations in a ring disk.

locations. Stresses are calculated at these and other radii using a finite difference method, and compared against a design stress that includes a safety margin for a specific material. If the calculated stress is above the design criteria, some disk thickness is increased sequentially in an iterative manner until the stress design criteria are satisfied at all radial stations.

A disk must be designed to withstand the centrifugal stresses generated by the rotating blades at the maximum rotational speed of the spool and to transmit the torque generated by the turbine blading to the appropriate compressor rotor. Only that portion of the disk inboard of the blade root is considered to carry the stresses, and this is referred to as the live disk. The parts of the disk between the blade roots (posts), as well as the blade roots themselves and the airfoils, constitute dead weight. Although it is the design of the live disk which is being addressed here, the posts must not be neglected when the total disk weight is quoted. The dead weight produces the pull stresses on the rim of the live disk, but as one moves inwards through the disk towards the bore, the disk must also support
Inner rim

Inner Shoulder

Bore

Centerline

Figure 3.-Radial stations and locations of design control calculations in a web disk.

an increasing proportion of the centrifugal stress generated by its own weight.

The average stress at the outer radius of the live disk is estimated by smearing the total centrifugal force of the dead weight around the circumference. The weight of the blades, including platforms and roots, and the axial chord at the root should already be known from the flowpath design. The radial location of the center of gravity of the combined dead weight must be known, however, in order to calculate the radial pull 


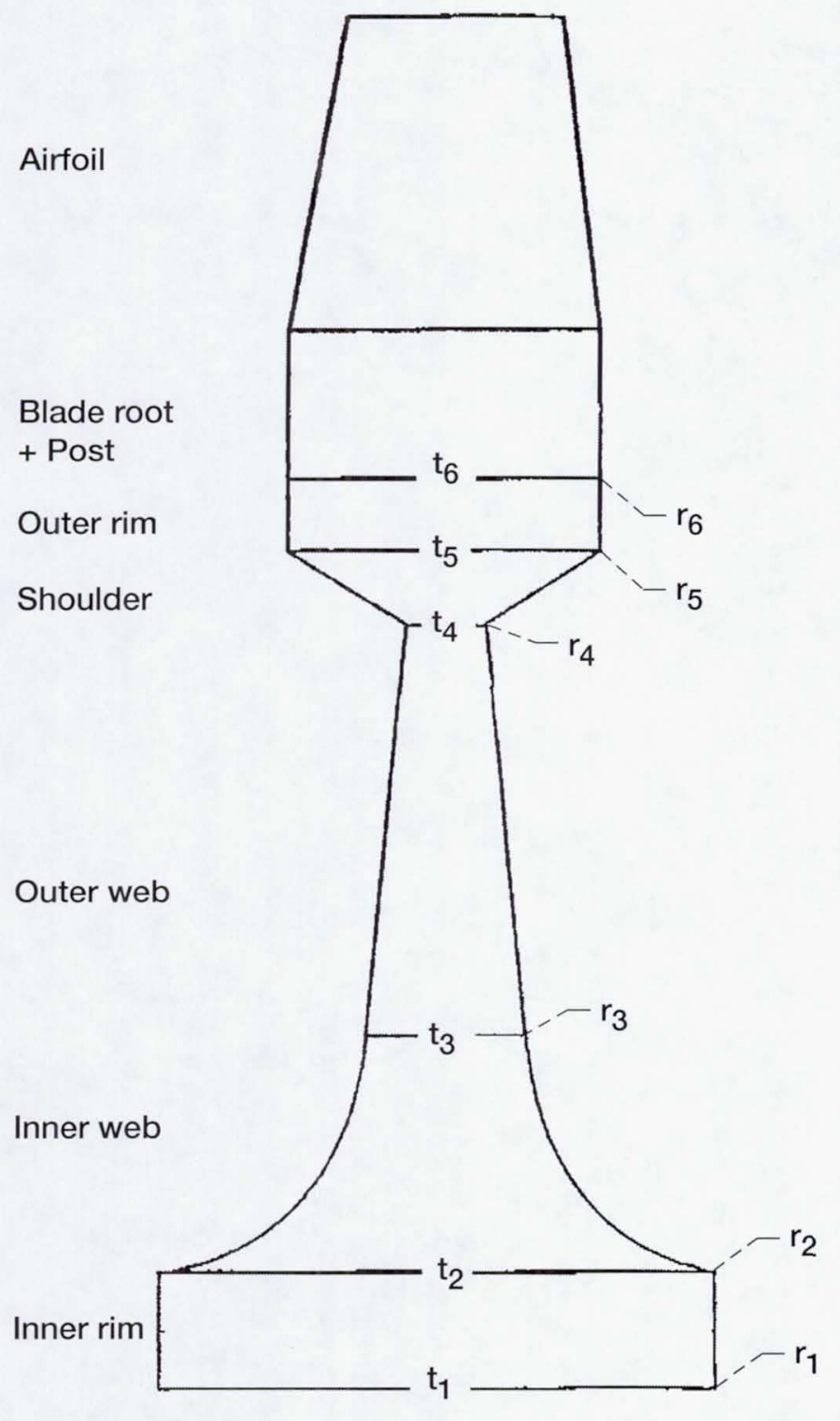

Bore

\section{Centerline}

\section{Figure 4.-Radial stations and locations of design control calculations in a hyperbolic disk.}

stress at the live rim, and consequently certain assumptions are made regarding the height and weight of the blade root, and the weight of the posts. The assumptions are as follows, where the term "total blade" refers to the "airfoil+platform+root".

For the ring disk:

Height of the blade root $=17.65 \%$ airfoil height

Weight of the blade root $=20 \%$ total blade weight

Weight of the post

$=10 \%$ total blade weight
For the web disk:

Height of the blade root $=25.00 \%$ airfoil height

Weight of the blade root $=20 \%$ total blade weight

Weight of the post $\quad=20 \%$ total blade weight

For the hyperbolic disk:

Height of the blade root $=53.85 \%$ airfoil height

Weight of the blade root $=50 \%$ total blade weight

Weight of the post $\quad=20 \%$ total blade weight

\section{DISK STRESS ANALYSIS}

The differential equation of equilibrium [2] takes the form:

$$
\frac{d}{d r}\left(t r \sigma_{r}\right)-t \sigma_{\theta}+t \rho \omega^{2} r^{2}=0
$$

where

$$
\begin{aligned}
& \sigma_{r}=\frac{E}{1-v^{2}}\left[\frac{d u}{d r}+v \frac{u}{r}-(1+v) \alpha T\right] \\
& \sigma_{\theta}=\frac{E}{1-v^{2}}\left[\frac{u}{r}+v \frac{d u}{d r}-(1+v) \alpha T\right]
\end{aligned}
$$

The radial steady-state temperature distribution for a disk is given by Fourier's law of conduction from [3]:

$$
T=T_{\text {bore }}+\frac{\Delta T}{\ln \left(\frac{r_{0}}{r_{i}}\right)} \ln \left(\frac{r}{r_{i}}\right)-T_{0}
$$

For a thin slice of disk material, the disk thickness, $t$, can be approximated by:

$$
\begin{gathered}
t=\mathrm{mr}+\mathrm{n} \\
m=\text { slope }=\frac{\Delta t}{\Delta r} \\
n=t-m \frac{\Delta t}{\Delta r}
\end{gathered}
$$

Substitute equations (2) to (4) and the disk thickness $t$ into equation (1) results in a single differential equation with one variable, displacement:

$$
\begin{array}{r}
\frac{d^{2} u}{d r^{2}}+\left(\frac{2 m r+n}{m r^{2}+n r}-\frac{v}{r}\right) \frac{d u}{d r}+\left[-\frac{v}{r^{2}}-\frac{1}{r^{2}}+\frac{v}{r}\left(\frac{2 m r+n}{m r^{2}+n r}\right)\right] u= \\
\alpha(1+v)\left[\frac{d T}{d r}+\left(\frac{2 m r+n}{m r^{2}+n r}-1\right) T\right]-\frac{\rho \omega^{2}\left(1-v^{2}\right)}{E} r
\end{array}
$$

Equation (5) is solved numerically using finite difference method with boundary conditions: 


$$
\sigma_{r}=0 \text { at } r=r_{i} ; \quad \text { thus, } \frac{d u}{d r}+v \frac{u}{r}-(1+v) \alpha T=0
$$

$$
\sigma_{r}=\text { rimstress at } r=r_{o} \text {; }
$$

$$
\text { thus, } \frac{d u}{d r}+v \frac{u}{r}-\frac{\text { rimstress } \cdot\left(1-v^{2}\right)}{E}-(1+v) \alpha T=0
$$

rimstress $=$ average centrifugal stress at the live rim

$$
=\frac{n_{b} m_{b} r_{c g}}{2 \pi r_{l} c} \omega^{2}
$$

The design criteria for the disk are:

$$
\left.\frac{\sigma_{y}}{s f . \sigma_{e}}-1.0>0 \quad \text { (design margin }\right)
$$

and

$$
\frac{0.47 \sigma_{u t s}}{\sigma_{\theta a v g}}-1.0>0 \quad \text { (disk burst criteria) }
$$

\section{DISK DESIGN PROCESS}

\section{Ring Disk}

The ring disk model is shown in figure 2. The design process for a ring disk consists of the following steps:

1. The thickness of the disk is set to the blade hub axial chord.

2. The lower limit for the bore radius, $r 1$, is calculated, based on the disk radius ratio provided in the input file and the airfoil hub radius. It should be noted that this will probably not be reached.

3. All the six thickness values are set to the value of the disk width in Step 1.

4. The radial extent of the disk from the outer live disk radius inwards is set initially to 0.1 inch.

5. The initial live disk height is divided into five equal sections to obtain radii $r 1$ through $r 6$.

6. The stress calculations are carried out and the design is checked for compliance with the design stress criteria (eqs. (6) and (7)).

7. If the design criteria are not satisfied at every radial location, the disk inner radius is reduced and Steps 5 and 6 are repeated.

8. If the design criteria are satisfied, disk weight is calculated and the disk design process is completed.

9. If the lower limit on bore radius (established in Step 2) is reached and the design criteria have still not been met, the program prints an appropriate message and stops.

\section{Web Disk}

The web disk model is shown in figure 3 . The design process for a web disk consists of the following steps:

1. The thickness of the disk outer rim is set to the blade hub axial chord.
2. The heights of the inner and outer rims and the inner and outer shoulders are specified by internal default values.

3. Minimum and maximum web and inner rim thickness are set by internal defaults.

4. Definition of the live disk geometry is initiated. The bore radius is fixed via the airfoil hub radius and the input value of disk radius ratio.

5. The six reference radii and thickness are set from the initial disk geometry.

6. The stress calculations are carried out and the design is checked for compliance with the design stress criteria (eqs. (6) and (7)).

7. If the design criteria are not satisfied at every radial location, the web thickness is held constant and the bore thickness is increased sequentially and Steps 5 and 6 are repeated. If the design criteria remain unsatisfied when the maximum limit on bore thickness is reached, the design process reverts to the minimum bore thickness, but the web thickness is increased, and Steps 4 to 6 are repeated.

8. If the design criteria are satisfied with neither the maximum bore thickness nor the maximum web thickness (established in Step 3) being exceeded, an acceptable web disk design is achieved and its weight is calculated. If the design criteria cannot be met within those limits, the program prints an appropriate message and stops.

\section{Hyperbolic Disk}

The hyperbolic disk model is shown in figure 4. The design process for a hyperbolic disk consists of the following steps:

1. The width of the disk outer rim is set to the hub axial chord.

2. The initial disk geometry is set up, which consists of the outer rim height, the shoulder height, the minimum outer web thickness, and the inner rim height. The maximum bore width and height are also established.

3. The six reference radii and thickness are set from the initial disk geometry.

4. Using a value of 1.0 for the disk shape factor, $d s f$, the inner portion of the initial disk geometry is defined, with the inner web thickness distribution being given by:

$$
\frac{t_{4}}{\left(r_{4}-r_{3}\right)}\left(r_{3}-r\right)^{d s f}
$$

5. The stress analysis routine is called and comparisons are made with the design stress criteria (eqs. (6) and (7)) at all the radial locations.

6. If the design criteria are not satisfied, the disk shape factor is increased and Steps 2 to 5 are repeated.

7. If the maximum limit on bore width is encountered before a satisfactory design is obtained, the value of the outer web thickness is increased, the disk shape factor is reset to 1.0, and Steps 2 to 5 are repeated.

8. If the design criteria are satisfied, disk weight is calculated and the disk design process is completed. 
9. A maximum limit for the outer web thickness is defined to be the outer rim thickness. A maximum limit for the disk shape factor is defined such that the inner web thickness does not exceed the bore thickness, which in turn is equal to one third of the live rim radius. If the maximum limits on outer web thickness and disk shape factor are both reached without a satisfactory disk design being achieved, the program prints an appropriate message and stops.

\section{Optimum Disk Design}

The code has an option to optimize the disk design. If this option is activated, the code will step through the design of ring, web, and hyperbolic disks sequentially. Provided that disks of each type are possible for the input conditions and geometry, the total weights will be accumulated. If it is not possible to design a disk of a particular type, the program will skip to the next configuration. The disk of minimum weight will finally be selected from the designs that are possible, and the corresponding output files will be generated.

To facilitate the optimization process, a self-adaptive numerical iteration scheme is used to solve equation (5). At every iteration, the step size is self-adjusted (increases or decreases) based on the differences in the design margin between the current and previous iterations. The self-adaptive numerical scheme greatly improves the computing efficiency of stress calculations, and enhances the process of disk-design optimization.

\section{TURBINE DISK LIFE ESTIMATION}

Using the disk-design methodology described in the previous section, the disks are designed at the sizing point of a flight cycle. The sizing point can be the on-design or an offdesign cycle condition. For a new engine design, the flight cycle is based on engineering judgment and input from the airframe manufacturer on its anticipated usage.

The basic mission life of a turbine disk is assumed to be governed by low cycle fatigue crack initiation due to major start-stop cycles. Life is assessed using the maximum strain range or stress range depending upon availability of information. Since any cracking in a disk is not acceptable, fatigue crack initiation is a sufficient criterion for the lifing calculations required herein. The equivalent Von-Mises strain or stress ranges at various critical radial locations are calculated and compared with existing experimental fatigue life curves tabulated for the various disk materials. When experimental fatigue results are unavailable, approximations of the fatigue resistance can be obtained with the aid of empirical correlations previously established between fatigue properties and corresponding tensile test properties. One such correlation that has seen widespread use for several decades is the Method of Universal Slopes (MUS) equation proposed by Manson [4] shown below.

$\Delta \varepsilon_{t}=\Delta \varepsilon_{e l}+\Delta \varepsilon_{i n}=\frac{3.5 \sigma_{u t s}}{E}\left(N_{f}\right)^{-0.12}+D^{0.6}\left(N_{f}\right)^{-0.60}$
The MUS equation has been used extensively, for example, in the design of the Space Shuttle Main Engines, by using a reduction factor of 3 on computed MUS life [5], i.e., $N_{f} / 3$.

It is recognized that actual disk lives depend upon countless details of a highly localized nature. Since such details cannot be known until a finalized design has been achieved and therefore cannot be accounted for during the earliest stages of design that is of concern herein.

\section{MATERIAL DATABASE}

A material database, consisting of the material data of most of the commonly-used aerospace materials, has been incorporated into WATE. These material data include density, modulus, yield and ultimate strengths, Poison's ratio, as well as some fatigue data. These data were mostly obtained from $[6,8]$. The list of materials is shown in Table 1 .

TABLE 1.- MATERIAL DATABASE
\begin{tabular}{|l|l|l|}
\hline Alloy 713C & Inconel-625 & N-155 \\
\hline Alloy 713LC & Inconel-690 & V-57 \\
\hline B-1900 & Inconel-706 & 4130 steel \\
\hline MAR-M247 & Inconel-718 & 4340 steel \\
\hline IN-100 & Rene 41 & 410 steel \\
\hline MAR-M302 & TD Nickel & $17-4$ PH steel \\
\hline MAR-M509 & Ti-6Al-4V & Udimet-500 \\
\hline WI-52 & Ti-17 & Udimet-700 \\
\hline Hastelloy-X & Ti-6-2-4-2 & Udimet-710 \\
\hline Hastelloy-S & Haynes-1 88 & Waspaloy \\
\hline Inconel-600 & L-605 & Rene-80 \\
\hline Inconel-601 & Alloy-901 & Rene-95 \\
\hline Inconel-617 & A-286 & \\
\hline
\end{tabular}

\section{OTHER ENHANCEMENTS OF WATE CODE}

In addition to the disk stress/life methodology and material database, the following capabilities are added to the WATE code:

1. Variation of disk type (ring, web, or hyperbolic) and material with compressor/turbine stages

2. Option to let user specify correlation for tip-speed versus 1 st stage pressure ratio for the compressor

3. Option to let user specify component length.

A controlling capability, developed by Boeing Aircrafts, has also been added to the WATE code. This provides an ability to control the position or dimensions of certain components using the inputs provided in WATE. By adding this capability, the time to generate a viable flowpath can be greatly reduced, since the need to iterate manually is no longer necessary. A control can be created for all the inputs that would be used to match one component to another. 


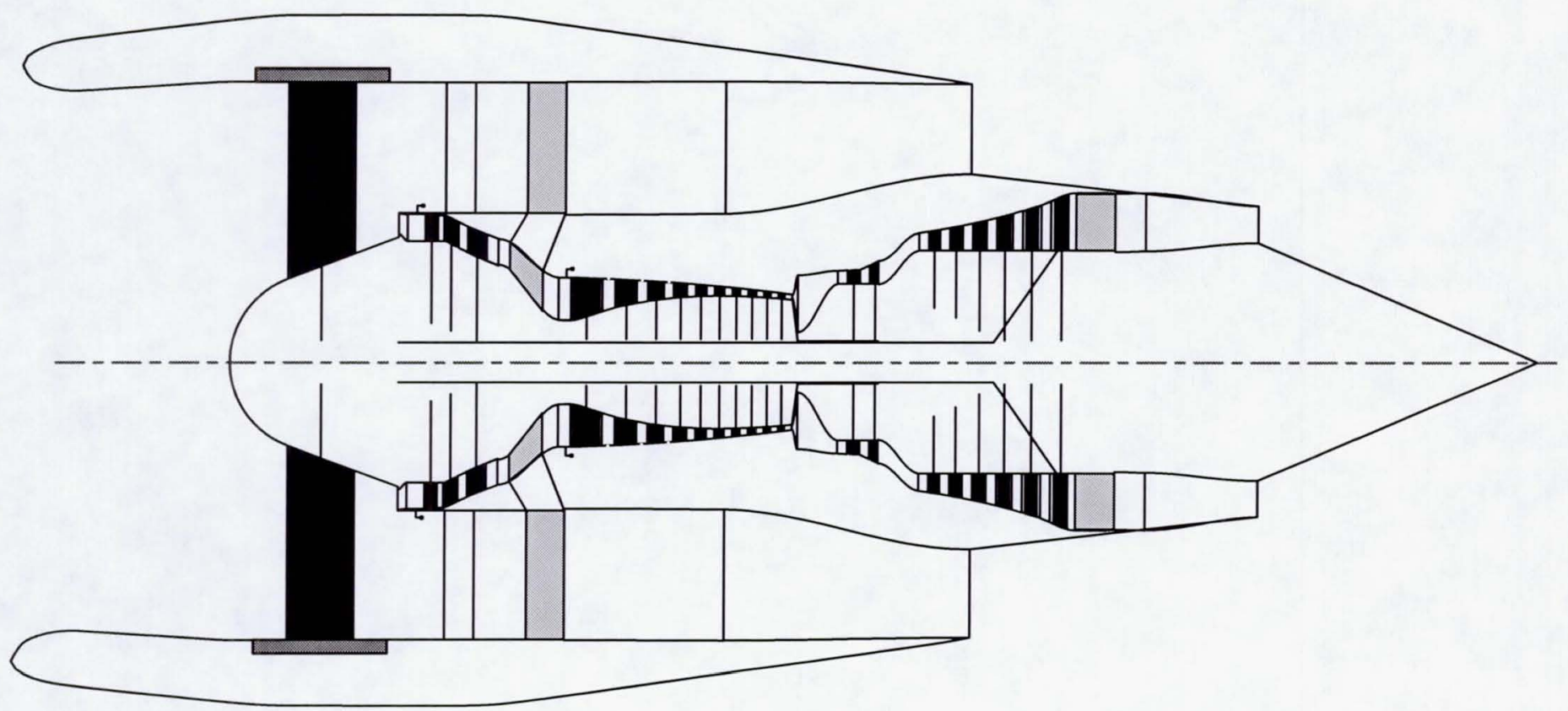

Figure 5.-Flowpath of a 300-passenger aircraft engine.

\section{APPLICATION OF THE COMPUTER CODE}

An engine sizing and weight estimation was performed on a 300-passenger aircraft engine (similar to a GE-90 engine), based on the thermodynamic parameters generated by an engine cycle analysis. The turbomachinery components and their materials are listed in Table 2.

TABLE 2.-MATERIALS FOR TURBOMACHINERY COMPONENTS

\begin{tabular}{|c|c|}
\hline $\begin{array}{l}\text { Fan: } \\
\text { blades } \\
\text { disk } \\
\text { disk type }\end{array}$ & $\begin{array}{l}\text { Ti } 6-4 \\
\text { Ti } 17 \\
\text { ring }\end{array}$ \\
\hline $\begin{array}{l}\text { LP compressor: } \\
\text { blades } \\
\text { disk } \\
\text { disk type }\end{array}$ & $\begin{array}{l}\text { Ti } 6-4 \\
\text { Ti } 17 \\
\text { ring }\end{array}$ \\
\hline $\begin{array}{l}\text { HP compressor: } \\
\text { blades } \\
\text { disk } \\
\text { disk type }\end{array}$ & $\begin{array}{l}\text { Ti } 6-4 \text { (stages } 1 \text { to } 6 \text { ) } \\
\text { Inconel-718 (stages } 7 \text { to } 10 \text { ) } \\
\text { Ti } 17 \text { (stages } 1 \text { to } 6 \text { ) } \\
\text { Inconel-718 (stages } 7 \text { to } 10 \text { ) } \\
\text { ring (stages } 1 \text { ) } \\
\text { web (stages } 2 \text { to } 10 \text { ) }\end{array}$ \\
\hline $\begin{array}{l}\text { HP turbine: } \\
\text { blades } \\
\text { disk type }\end{array}$ & $\begin{array}{l}\text { Rene- } 80 \\
\text { hyperbolic }\end{array}$ \\
\hline $\begin{array}{l}\text { LP turbine: } \\
\text { blades } \\
\text { disk type }\end{array}$ & $\begin{array}{l}\text { Rene- } 80 \\
\text { web }\end{array}$ \\
\hline
\end{tabular}

For the turbine disk materials, three different alloys were assessed and their fatigue lives compared:

HP and LP turbine disks:

The results:

Total engine dry wt. $(\mathrm{kg})$ :

Total engine weight $(\mathrm{kg})$ :

Total HP and LP disk wt. ( $\mathrm{kg})$ :

Minimum turbine (HPT) disk

life (flight cycles):
Engine A Engine B Engine C Inconel-718 Rene-95 Rene-88*

$\begin{array}{rrr}7537 & 7461 & 7580 \\ 10182 & 10105 & 10226 \\ & & \\ 989 & 900 & 1034 \\ 3292 & 6000 & >30000\end{array}$

*Note: based on the limited data published by Huron and Roth [9].

The results show that although Engine $\mathrm{C}$ is the heaviest among the three engines, it has the longest turbine disk life. In comparison, the dry weight of a GE-90 engine is $7559 \mathrm{~kg}$ [10]. Typical commercial production engines require 15,000 to 20,000 flight cycles in the hot section components to satisfy customer and manufacturer requirements. The enhanced WATE code provides additional insight into the design trade-off between engine life and engine weight. Flowpath of Engine $\mathrm{C}$ is shown in figure 5 .

\section{CONCLUSIONS}

The current enhancements, based on material database and structural analysis, is a major improvement over most existing preliminary design codes, which usually either do not consider structural integrity per se, or merely rely on general empirical guidelines. Collectively, these enhancements provide a more realistic and systematic way to estimate the engine size and 
weight at the preliminary design stage. They also provide additional insight into the design trade-off between engine life and engine weight. Since materials and weight can affect engine cost, the current effort paves the way for an automated engine design tool, which would allow engine developers to easily perform design trade-offs between engine performance, durability, and cost.

\section{ACKNOWLEDGMENTS}

The authors wish to acknowledge the efforts of Dr. Gary R. Halford of NASA Glenn Research Center in providing an insight in the fatigue lifing used in this study.

\section{REFERENCES}

[1] Onat, E. and Klees, G.W., 1979, "A Method to Estimate Weight and Dimensions of Large and Small Gas Turbine Engines," NASA CR-159481.

[2] Ugural, A.C. and Fenster, S.K., 1987, Advanced Strength and Applied Elasticity, Second Edition, Elsevier Science Publishing Co., Inc.

[3] Holman, J.P., 1990, Heat Transfer, Seventh Edition, McGraw-Hill Book Company.
[4] Manson, S.S., 1965, "Fatigue: A Complex Subject-Some Simple Approximations, Experimental Mechanics," 5, No. 7, pp. 193-226.

[5] Newell, J.F., 1994, "A Note of Appreciation for the MUS," PVP, 290, Material Durability/Life Prediction Modeling: Materials for the 21st Century, ASME, NYC, pp. 57-58.

[6] Aerospace Structural Metals Handbook, 1998 Edition.

[7] ASM International: Metals Handbook, Tenth Edition, 1990.

[8] ASM International: Materials Properties HandbookTitanium Alloys, 1994.

[9] Huron, E.S. and Roth, P.G., 1996, "The Influence of Inclusions on Low Cycle Fatigue Life in a P/M Nickel-Base Disk Superalloy," Superalloys.

[10] Jane's Aircraft Engine, Jane's Information Group Ltd, 1997. 